\title{
Design Simulation and Performance Assessment of Improved Channel Estimation for Millimeter Wave Massive MIMO Systems
}

\author{
Aditi Sharma $^{a}$, Ashish Kumar Sharma ${ }^{\text {b Laxmi Narayan Balai }}{ }^{c}$ \\ ${ }^{a}$ M.Tech. Scholar, Department of Electronics \& Communication Engineering, YIT, Jaipur, India \\ ${ }^{b}$ Assistant Professor, Department of Electronics \& Communication Engineering, YIT, Jaipur, India \\ ${ }^{c}$ HOD, Department of Electronics \& Communication Engineering, YIT, Jaipur, India
}

\begin{abstract}
In this paper, we have optimize specificities with the use of massive MIMO in $5 \mathrm{G}$ systems. Massive MIMO uses a large number, low cost and low power antennas at the base stations. These antennas provide benefit such as improved spectrum performance, which allows the base station to serve more users, reduced latency due to reduced fading power consumption and much more. By employing the lens antenna array, beam space MIMO can utilize beam selection to reduce the number of required RF chains in $\mathrm{mm}$ Wave massive MIMO systems without obvious performance loss. However, to achieve the capacity-approaching performance, beam selection requires the accurate information of beam space channel of large size, which is challenging, especially when the number of RF chains is limited. To solve this problem, in this paper we propose a reliable support detection (SD)-based channel estimation scheme. In this work we first design an adaptive selecting network for mm-wave massive MIMO systems with lens antenna array, and based on this network, we further formulate the beam space channel estimation problem as a sparse signal recovery problem. Then, by fully utilizing the structural characteristics of the mm-wave beam space channel, we propose a support detection (SD)-based channel estimation scheme with reliable performance and low pilot overhead. Finally, the performance and complexity analyses are provided to prove that the proposed SD-based channel estimation scheme can estimate the support of sparse beam space channel with comparable or higher accuracy than conventional schemes. Simulation results verify that the proposed SD-based channel estimation scheme outperforms conventional schemes and enjoys satisfying accuracy even in the low SNR region as the structural characteristics of beam space channel can be exploited.
\end{abstract}

Keywords: 5G, MIMO, Massive MIMO, SD-Based Channel

\section{INTRODUCTION}

In recent years the need for wireless power has increased exponentially, with the number of wireless devices and new smartphone users growing. In order to increase the performance it is important to increase bandwidth or spectral efficiency. Since increasing the bandwidth is an expensive factor, spectral effectiveness must be considered. The transmission and receiver can be supplemented by several antennas. Multiple input multiple output antennas (MIMO) increase both communication efficiency and communication capability (by transmitting data in separate antennas). In general MIMO systems have two classes: MIMO point-to point systems and -MIMO (MU-MIMO) multi-user systems. The transmitter as well as the recipient are fitted with many antennas on point-to - point MIMO.

MIMO systems have been integrated into many wireless standards of new generation, such as advanced LTE, wireless
LAN, etc. Interference between co channel users is the major challenge in the MU-MIMO method. Complex methods must also be used to minimise interference with the co-channel. The MU-MIMO system is known as the Massive MIMO system or large MU-MIMO system antenna arrays, with a hundred of antenna systems in the BS which serve several antenna user terminals at the same time at same frequency and time. The huge MIMO device in which BS has a large number of antennas and serves several single user terminals is one of the architectures proposed for $5 \mathrm{G}$ wireless communication.

One of the architectures proposed for $5 \mathrm{G}$ wireless communication is the massive MIMO system in which BS is equipped with a large number of antenna and serves multiple single antenna user. Massive MIMO is an antenna technology for $5 \mathrm{G}$ and beyond future-oriented networks. 
At $5 \mathrm{G}$, even if our mobile phone is in one bar or two, or we're thinking about video chatting and streaming blockbuster movies, we can't even think of downloaded a file twice. Often, it is as seamless as streaming music to conduct such practices. 5 G NR (New Radio) technology is one of the key elements to activate these $5 \mathrm{G}$ user interactions. Multiple Input Multiple output ( MIMO) technology and with the global rollout of $5 \mathrm{G}$, consumer aspirations also grow as the capacities of today's mobile networks significantly increase. We have covered mm Wave's concepts for mobile, beam shaping and low latency in our ongoing series of blog postings to illustrate our breakneck innovations that make 5 $\mathrm{G}$ a reality. Now we can view huge $5 \mathrm{G}$ NR MIMO and how this technology improves mobile device users as well as networks. Huge MIMO is the secret to allowing very high data speeds of $5 \mathrm{G}$ and aims to increase the capacity of $5 \mathrm{G}$ to a new stage.

\section{LITERATURE REVIEW}

[Hengtao He et.al, 2018] The channel calculation is very difficult because the recipient has an ampl chain (mmWave) mm-wave (MF) in multiple input and multiple output systems. We use an approximate message centred on the denoising network of moving networks (LDAMP) in order to solve this problem. The research framework also provides the asymptotic output of LDAMP on the channel evaluation. The results of the analyses and simulations are from LDAMP. With few RF chains the network reaches advanced compressed sensing (CS). [1]

[Shiguo Wang et.al, 2019] discussed Huge multiple input output is a big technology in $5 \mathrm{G}$, It allows many users to use pre-coding or beam forming techniques in the same frequency block, thereby increasing power, reliability and efficiency in energy. A key issue in a large MIMO is the power allocation for each antennas to achieve a specific goal, i.e. to optimize the minimum user energy. We are limited to sub $6 \mathrm{GHz}$ networks based on the Time-Division (TDD). Numeric results show that the results of a widely used heuristic based on the bisectional algorithm are very approximated by our DNN-based method. [2]

[Yu Zhao et.al, 2020] The author says the combination of cell-free multi-input (MIMO) systems with a microwave (mmWave) tape is indeed one of the most promising technical enablers to the imagined wireless Gbit / s experience. The author says. However, both massive antennas and broad bandwidth at mmWave trigger high computer complexity to use a precise approximation of the channel state information. With the sparse channel matrix of the mmWave being a natural image, we propose a realistic and accurate channel estimation method on the basis of the fast and versatile FFDNet. [3]
[Yu Jin et.al, 2019] Wireless networks are complex, huge and demanding on capacity increased demand led to difficulties in network component management and monitoring. Smart data-driven designs and methods would also be required to reform the 5 th generation $(5 \mathrm{G}$ ) of mobile networks for self-organization. So mathematical models have been developed and adapted between modems in the last decade. This article offers a full overview of recent research into in-depth models of learning for strong MIMO systems. The main part of the work includes the reconstruction of the traditional communication system using deep learning models. [4]

[Vandana Bhatia et.al, 2020] In this paper, the author briefly describes how the calculation of the multiple input channel (MIMO) for vehicle communication is highly demanding because of the shift in channel and low latency. In this paper, the newly emerging and popular deep neural network is used to improve the accuracy and reduce the delay of massive MIMO channel evaluation to learn the sparing information in the MIMO channel and to more reliably and more rapidly estimate the channel. [5]

\section{OBJECTIVES \& PROPOSED METHODOLOGY}

\subsection{Objectives}

The main goal of the thesis is to provide efficient estimation of channels and precoding for a massive MIMO network.

- To achieve the goal, the following work steps are considered as objectives: To create a Massive MIMO Communication System Model and simulate it.

- To investigate the problems of Massive MIMO system channel estimation and precoding. In mmwave huge, we examine the beam space channel estimation problem. With a lens antenna array, MIMO systems are possible.

- We first create an adaptive selection network for mm-wave huge MIMO systems with lens antenna arrays, and then build a system around it. The beam space channel estimation problem is therefore reformulated as a sparse signal recovery problem.

- The structural properties of the mm-wave beam space channel are then completely used. We present a channel estimate approach based on support detection (SD) that is both reliable and low in pilot overhead.

- Finally, performance and complexity analyses show that the proposed SD-based channel estimation scheme can estimate the support of sparse 
beam space channels with equivalent or higher accuracy than existing techniques.

\subsection{Proposed Methodology}

We present a support detection (SD)-based channel estimation approach that uses CS tools to estimate the beam space channel with reduced pilot overhead in this study.

1) The newly-proposed selecting network in mm-wave MIMO is designed to replace an adapted selecting network consisting of a small number of one-bit phase shifters. With regard to data transmission, the suggested adaptive selection network may pick beams such as the conventional one, and can be used as a combiner for channel estimate to achieve efficient beam space channel measurements. Then we formulate the Beam space channel estimation problem as a sparse signal recuperation issue based on the proposed adaptive selection network.

2) We are proposing an estimation approach for SD channels. The essential idea is to partition the whole problem of the estimated channel into a series of sub-problems, each of which takes one sparse channel component into account. For every channel component, we initially determine its support by using the structural properties of the mm-wave beam space channel (e.g. the index set of non-zero items in a sparse vector). Then, the influence of the channel component is removed and a similar procedure is used to detect support of the following channel component. The big beam space channel can be estimated with modest pilot overhead after the supports of all channel components have been detected.

3) We prove to be able to detect channel component supported more accurately than conventional CS algorithms with the suggested SD-based channel estimating scheme. Analysis of complexity reveals that the SD channel estimate is also quite low. Simulated results verify, even in the low signal to noise (SNR) region that the SD-based channel estimates are of satisfactory precise and low pilot overhead.

\section{RESULT ANALYSIS}

In this work, we shall show that we can detect the support more precisely than the standard CS algorithms in the proposed SD-based channel estimate. It may be observed that estimating the position of the stronger element determines the accuracy of the detected support. We have therefore simulated the suggested approach in detail to evaluate the accuracy of the discovered support.

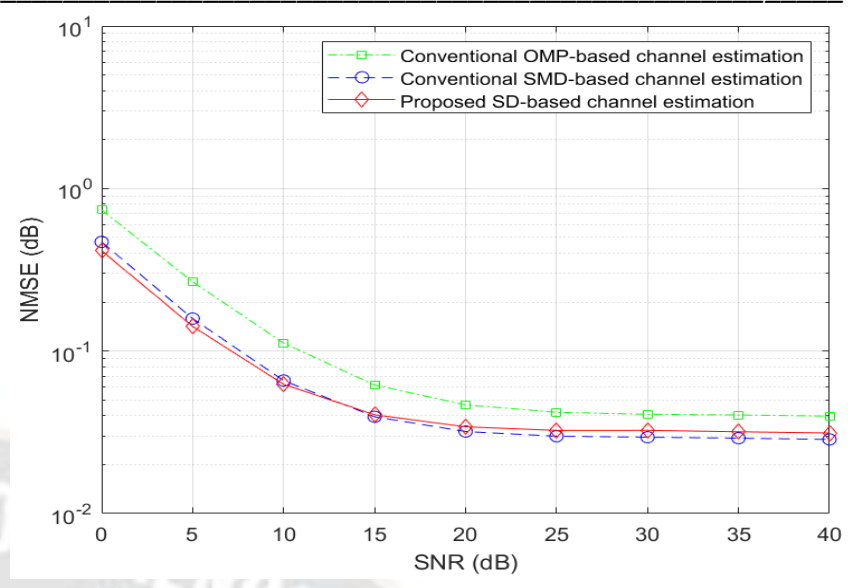

Figure 1 NMSE Analysis of Proposed Algorithms with Comparative Assessment

Figure 1 shows first that the suggested SD-based channel estimate of $Q=96$ instants is likely to generate NMSE results close to the SMD-based channel estimate at $\mathrm{Q}=\mathrm{N}=256$ instants. Furthermore, we also note that the SD channel estimate is more precise than the OMP channel estimate where the uplink SNR is low (e.g., less than $15 \mathrm{~dB}$ ). The performance gap is less when the uplink SNR is large. This can be explained by the fact that the OMP-based channel estimate can also accurately estimate the support of the beamspace channel when the UPLS SNR is high and the total number of instants $Q$ is large enough however, because to the small transmission power of the users, the uplink SNR is frequently low. We can therefore infer that the SD channel estimation scheme that has been proposed is attractive. Lastly, it indicates that the NMSE efficiency of all investigated canal estimates is saturated when the uplink SNR is high enough. This is because while beam space channel's non-zero elements may be estimated reliably with high enough uplink SNR, the mistake of small-powered elements as zero does not disappear.

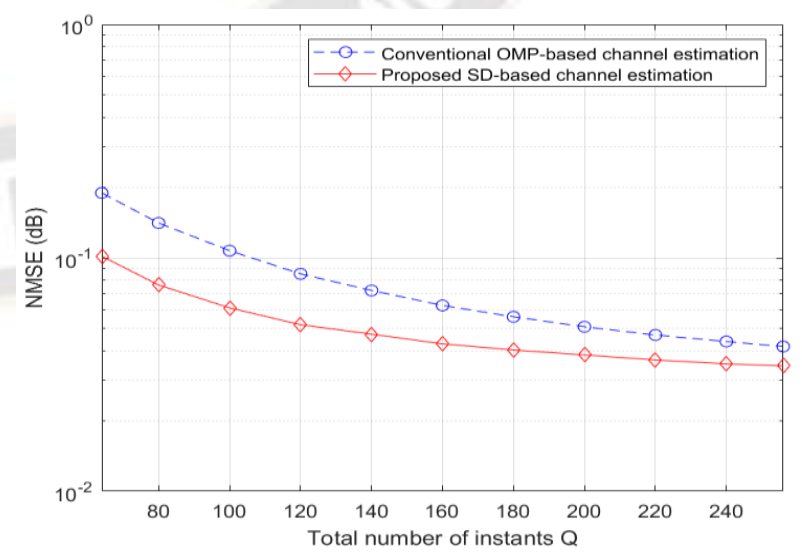

Figure 2 NMSE Analysis of Proposed Algorithms with Comparative Assessment With Respect to Instants

Figure 2 displays a comparison of NMSE performance with the total number of Q instants where the SNR uplink is set to 
$10 \mathrm{~dB}$. In above, the total number of Q-instants required by the SD channel estimation is significantly smaller than the OMP channel estimate to attain the same exactness. The total number of instants needed for OMP-based channel estimates is $\mathrm{Q}=190$, for example, in order to attain the NMSE of 5 | 10-002, the proposed estimate for the SD-based channel requires $\mathrm{Q}=120$ instants. Moreover, given that in SMD channel assessments the number of $\mathrm{Q}$ instants required is larger than $\mathrm{N}$ (e.g., $\mathrm{Q}=\mathrm{N}=256$ ), it may also be concluded that the SD channel estimating scheme suggested can deliver sufficient performance with additionally lowered overhead pilots.

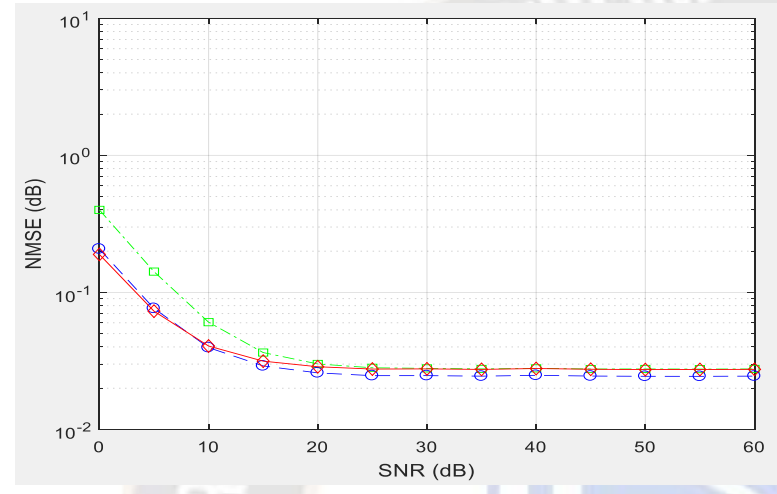

Figure 3 NMSE Analysis of Proposed Algorithms with Comparative Assessment

(Case-2, 512 Transmit Antennas, 32 Number of Users and Upto $60 \mathrm{~dB}$ SNR)

Figure 3 presents the performance comparison of the Normalized Average Square Error (NMSE) between SMDbased, OMP-based and SD-based estimate channel schemes. The performance has been analysed with parameter variation to test the application of simulated system. Figure 3 proves that the performance of the system is optimum for wide range of SNR. The number of transmit antennas has been increased along with the increament in the number of users. The performance analysis of the NMSE characteristics proves the applicability of the proposed system.

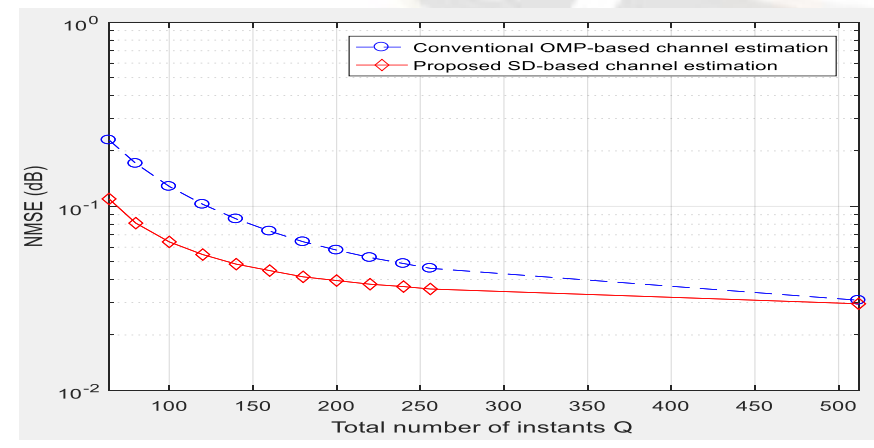

Figure 4 NMSE Analysis of Proposed Algorithms with Comparative Assessment with Respect to Instants (Case-2, 512 Transmit Antennas, 32 Number of Users and Upto 512 instants)
Figure 4.4 displays a comparison of NMSE performance with the total number of Q instants where the SNR uplink is set to $10 \mathrm{~dB}$. It can be observed that the total number of Q-instants required by the SD channel estimation is significantly smaller than the OMP channel estimate to attain the same exactness. Moreover, given that in SMD channel assessments the number of $\mathrm{Q}$ instants required is larger than $\mathrm{N}$ (e.g., $\mathrm{Q}=\mathrm{N}=$ 512), it may also be concluded that the SD channel estimating scheme suggested can deliver sufficient performance with additionally lowered overhead pilots.

\section{CONCLUSION}

Problem with the channel estimation for MIMO lens antenna array huge mm-wave systems. Especially in order to approach the beam space channel assessment problem as a sparse signal recovery problem, we first develop an adaptive selector network for MIMO huge mm-wave system with a lens antenna array. Then we present an SD-based channel estimate scheme with reduced pilot overhead, based on the specific structural characteristics of the mm-wave pool channel. The performance analysis shows that the proposed SD based channel estimation system allows slim beam space channel support to be detected with greater precision than standard CS techniques. The complexity research demonstrates further that the SD channel estimate is lowly complex, comparable to that of the LS algorithm. Simulations verify that even within low SNR region, NMSE performance may be significantly improved by the proposed SD-based channel estimates method than standard schemes. For MIMO huge MIMO systems with a lens antenna array, this makes them more appealing. We will enhance the suggested SD channel estimate scheme to include instances when customers use multiple antennas in our future work.

\section{REFERENCES}

[1] He, Hengtao, Chao-Kai Wen, Shi Jin, and Geoffrey Ye Li. "Deep learning-based channel estimation for beamspace mmWave massive MIMO systems." IEEE Wireless Communications Letters 7, no. 5 (2018): 852-855.

[2] Wang, Shiguo, Lifang Li, Rukhsana Ruby, and Peng Li. "A general hybrid precoding scheme for millimeter wave massive MIMO systems." Wireless Networks 26, no. 2 (2020): 1331-1345.

[3] Zhao, Yu, Ignas G. Niemegeers, and Sonia Heemstra De Groot. "Power Allocation in Cell-Free Massive MIMO: A Deep Learning Method." IEEE Access 8 (2020): 87185-87200.

[4] Jin, Yu, Jiayi Zhang, Shi Jin, and Bo Ai. "Channel estimation for cell-free mmWave massive MIMO 
through deep learning." IEEE Transactions on Vehicular Technology 68, no. 10 (2019): 1032510329..

[5] Bhatia, Vandana, Malay Ranjan Tripathy, and Priya Ranjan. "Deep Learning for massive MIMO: Challenges and Future prospects." In 2020 IEEE 9th International Conference on Communication Systems and Network Technologies (CSNT), pp. 26-31. IEEE, 2020.

[6] Huang, Xiao, and Sicong Liu. "Massive MIMO Channel Estimation for Vehicular Communications: A Deep Learning Based Approach." In 2020 IEEE International Conference on Communications Workshops (ICC Workshops), pp. 1-6. IEEE, 2020.

[7] Liao, Yong, Yuanxiao Hua, and Yunlong Cai. "Deep Learning Based Channel Estimation Algorithm for Fast Time-Varying MIMO-OFDM Systems." IEEE Communications Letters 24, no. 3 (2019): 572-576.

[8] Ye, Hao, Geoffrey Ye Li, and Biing-Hwang Juang. "Power of deep learning for channel estimation and signal detection in OFDM systems." IEEE Wireless Communications Letters 7, no. 1 (2017): 114-117.

[9] Yang, Qianqian, Mahdi Boloursaz Mashhadi, and Deniz Gündüz. "Deep convolutional compression for massive MIMO CSI feedback." In 2019 IEEE 29th international workshop on machine learning for signal processing (MLSP), pp. 1-6. IEEE, 2019.

[10] Qiang, Hu, Gao Feifei, Zhang Hao, Jin Shi, and Li Geoffrey Ye. "Deep Learning for MIMO Channel Estimation: Interpretation, Performance, and Comparison." arXiv preprint arXiv:1911.01918 (2019).

[11] Mei, Kai, Jun Liu, Xiaochen Zhang, and Jibo Wei. "Machine Learning Based Channel Estimation: A Computational Approach for Universal Channel Conditions." arXiv preprint arXiv:1911.03886 (2019).

[12] Ma, Xisuo, and Zhen Gao. "Data-Driven Deep Learning to Design Pilot and Channel Estimator for Massive MIMO." IEEE Transactions on Vehicular Technology 69, no. 5 (2020): 5677-5682.

[13] Yuan, Jide, Hien Quoc Ngo, and Michail Matthaiou. "Machine learning-based channel estimation in massive MIMO with channel aging." In 2019 IEEE 20th International Workshop on Signal Processing Advances in Wireless Communications (SPAWC), pp. 1-5. IEEE, 2019.

[14] Balevi, Eren, Akash Doshi, and Jeffrey G. Andrews. "Massive mimo channel estimation with an untrained deep neural network." IEEE Transactions on Wireless Communications 19, no. 3 (2020): 2079-2090.

[15] Boloursaz Mashhadi, Mahdi, and Deniz Gündüz. "Deep Learning for Massive MIMO Channel State
Acquisition and Feedback." Journal of the Indian Institute of Science (2020): 1-14.

[16] Motade, Sumitra N., and Anju V. Kulkarni. "Channel estimation and data detection using machine learning for MIMO 5G communication systems in fading channel." Technologies 6, no. 3 (2018): 72.

[17] Albataineh, Zaid, Khaled Hayajneh, Haythem Bany Salameh, Chinh Dang, and Ahmad Dagmseh. "Robust Massive MIMO Channel Estimation for 5G Networks Using Compressive Sensing Technique." AEUInternational Journal of Electronics and Communications (2020): 153197.

[18] Dörner, Sebastian, Sebastian Cammerer, Jakob Hoydis, and Stephan Ten Brink. "Deep learning based communication over the air." IEEE Journal of Selected Topics in Signal Processing 12, no. 1 (2017): 132-143.

[19] Ma, Wenyan, Chenhao Qi, Zaichen Zhang, and Julian Cheng. "Sparse channel estimation and hybrid precoding using deep learning for millimeter wave massive MIMO." IEEE Transactions on Communications 68, no. 5 (2020): 2838-2849.

[20] Yang, Qianqian, Mahdi Boloursaz Mashhadi, and Deniz Gunduz. "Distributed Deep Convolutional Compression for Massive MIMO CSI Feedback." arXiv preprint arXiv:2003.04684 (2020).

[21] Neumann, David, Thomas Wiese, and Wolfgang Utschick. "Learning the MMSE channel estimator." IEEE Transactions on Signal Processing 66, no. 11 (2018): 2905-2917.

[22] Mashhadi, Mahdi Boloursaz, and Deniz Gunduz. "Pruning the Pilots: Deep Learning-Based Pilot Design and Channel Estimation for MIMO-OFDM Systems." arXiv preprint arXiv:2006.11796 (2020).

[23] Simeone, Osvaldo. "A very brief introduction to machine learning with applications to communication systems." IEEE Transactions on Cognitive Communications and Networking 4, no. 4 (2018): 648-664.

[24] Soltani, Mehran, Vahid Pourahmadi, Ali Mirzaei, and Hamid Sheikhzadeh. "Deep learning-based channel estimation." IEEE Communications Letters 23, no. 4 (2019): 652-655.

[25] Verenzuela, Daniel, Emil Björnson, Xiaojie Wang, Maximilian Arnold, and Stephan ten Brink. "MassiveMIMO Iterative Channel Estimation and Decoding (MICED) in the Uplink." IEEE Transactions on Communications 68, no. 2 (2019): 854-870.

[26] Yang, Yuwen, Feifei Gao, Xiaoli Ma, and Shun Zhang. "Deep learning-based channel estimation for doubly selective fading channels." IEEE Access 7 (2019): 36579-36589. 
[27] Ye, Hongyuan, Feifei Gao, Jing Qian, Hao Wang, and Geoffrey Ye Li. "Deep learning based denoise network for CSI feedback in FDD massive MIMO systems." IEEE Communications Letters (2020).

[28] Yuan, Jide, Hien Quoc Ngo, and Michail Matthaiou. "Machine learning-based channel estimation in massive MIMO with channel aging." In 2019 IEEE 20th International Workshop on Signal Processing Advances in Wireless Communications (SPAWC), pp. 1-5. IEEE, 2019.

[29] Ngo, Hien Quoc. Massive MIMO: Fundamentals and system designs. Vol. 1642. Linköping University Electronic Press, 2015.

[30] T. L. Marzetta, "Massive MIMO: An Introduction," in Bell Labs Technical Journal, vol. 20, pp. 11-22, 2015, doi: 10.15325/BLTJ.2015.2407793.

[31] Hassan, Noha, and Xavier Fernando. "Massive MIMO wireless networks: An overview." Electronics 6, no. 3 (2017): 63.

[32] T. Sanguankotchakorn and C. A. Kumar, "Optimizing Power by Combining Small Cells with a Massive MIMO for Maximum Energy Efficiency Under Two Path Loss Models," 2019 International Conference on Information Networking (ICOIN), Kuala Lumpur, Malaysia, 2019, pp. 125-130, doi: 10.1109/ICOIN.2019.8718152.

[33] E. Björnson, M. Kountouris and M. Debbah, "Massive MIMO and small cells: Improving energy efficiency by optimal soft-cell coordination," ICT 2013, Casablanca, Morocco, 2013, pp. 1-5, doi: 10.1109/ICTEL.2013.6632074. 